\title{
Technical Note: \\ The Development of Extradosed Bridge Concept from a Vision to a Reality
}

\author{
Theryo, T.S. ${ }^{1}$
}

\begin{abstract}
The development of Extradosed bridge from a vision to a real project is presented in this paper. Mathivat of France who is recognized as the inventor of this bridge type, envisioned a new bridge type suitable for medium span ranges in 1979. He suggested that some of the cantilever tendons in balanced cantilever bridge can be deviated to the top deck supported by short tower resemble stay cables. The structural benefit of this idea is increasing positive moment capacity by increasing eccentricity over the pier and enhanced shear capacity. Therefore, with the same conditions, longer span can be designed. Due to small angle of stay cables, the cable allowable stress at serviceability is about the same level with balanced cantilever bridge and no significant penalty on fatigue resistance unlike stay cables for conventional cable stayed bridges. The Extradosed bridge offers economic benefit for medium span range and aesthetically pleasing.
\end{abstract}

Keywords: Concrete medium-span bridge; Extradosed; balanced cantilever.

\section{Introduction}

Jacques Mathivat of France in his book "The Cantilever Construction of Prestressed Concrete Bridges" in 1979 [1],_envisioned a potential new type of bridge which is a transition from a post-tensioned balanced cantilever bridge to cable stayed bridge. In other word, a new bridge type which hybrid of post-tensioned balanced cantilever bridge and a cable stayed bridge. At that time, both balanced cantilever bridge and cable stayed bridge had been well established as two distinct type of bridges with its own specific structural behavior, such as economic benefit, construction method and span length limitations. Mathivat was aware that the economical span ranges of balanced cantilever bridge between $50 \mathrm{~m}$ (164 feet) to $150 \mathrm{~m}$ (500 ft.), while cable stayed bridge span range was over $200 \mathrm{~m}$ (650 ft.) at that time. Keep in mind that the economical span ranges mentioned above may have changed nowadays due to advancement in the design, material, and construction methods. It is suggested that economical span ranges for Extradosed bridge is between $60 \mathrm{~m}$ (200 ft.) to 275 m (900 ft.) [2] Cantilevering construction method for a long span bridge either post-tensioned box girder balanced cantilever method bridges or cable stayed bridge is still the dominance construction method.

\footnotetext{
${ }^{1}$ Major Bridge Design Engineer with Florida Department of Transportation, Tallahassee, FL, USA

Director of ICESNA, USA.

Email: theryott@gmail.com
}

Note: Discussion is expected before July, $1^{\text {st }} 2021$, and will be published in the "Civil Engineering Dimension", volume 23, number 2, September 2021.

Received 01 February 2021; revised 06 February 2021; accepted 08 February 2021.
In balanced cantilever construction method, negative bending moment during construction is the primary forces, the structural engineer must overcomes primarily by cantilever tendons as shown in Figure 1.

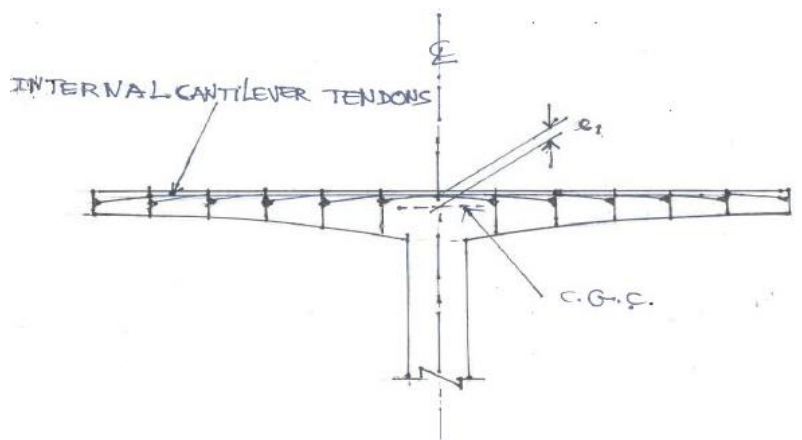

Figure 1. Typical Balanced Cantilever Bridge

The balanced cantilever bridge has its own drawbacks, since the longer the span the dead loads become larger and prohibitive to build. To increase the bending moment capacity at the pier, one should increase the number of cantilever tendons or eccentricity by increasing structural depth. Adding more cantilever tendons is limited by the spaces available in the top flange of a box girder. Mathivat saw the opportunity to increase bending moment and shear capacity by partially deviating some of the cantilever tendons over the pier (critical moment and shear) above the top flange to become external tendons supported by short deviator/tower as shown in Figure 2. This brilliant solution will be able to take the advantages of both internal cantilever and external tendons with larger eccentricity. Therefore, cantilever tendons can be reduced or extend longer span. As can be observed in Figures 1 and 2, the tendon eccentricity $\mathrm{e}_{2} \gg \mathrm{e}_{1}$. 


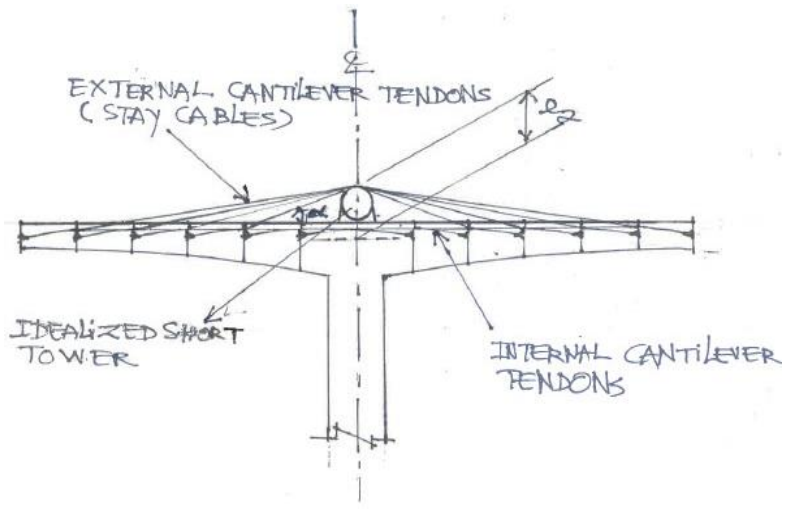

Figure 2. Balanced Cantilever Bridge with Deviated External Tendons over the Pier

In addition, Mathivat proposed higher allowable stress ( 0.6 of the ultimate tensile strength (fpu)) for the external tendons/short stays than conventional stay cables (0.4 fpu-0.45 fpu) at Service Limit State design load combinations. According to Mathivat, this type of bridge would be economical in the transition span ranges between conventional balanced cantilever and cable stayed bridges.

In 1988 Mathivat proposed the above idea in a bridge competition in France for Autoroute A64 viaduct across Arret-Darre as shown in Figure 3. He called the new bridge concept Extradosed. Extradosed comes from "extrados" which means external or the upper curve of an arch. The new bridge concept was published in FIP Journal: Recent Development in Prestressed Concrete bridges [3]. Although the proposed bridge was not built, however the new concept has inspired many bridge engineers around the world. The first Extradosed bridge built in France is Saint-Remy Bridge built in 1996.

It is important to note that in 1980, Christian Menn of Switzerland designed Ganter Bridge in Switzerland as shown in Figure 4. The structural concept of Ganter Bridge is very similar to the Extradosed Bridge, except the stay cables are internal tendons and cast inside a concrete wall. There are opinions that Ganter Bridge should also considered as an Extradosed Bridge. One of the obvious structural behavior differences is the stay cables as discussed in the followings. This type of bridge is commonly called Finback Bridge in the US or Sail bridge somewhere else. It is the author's opinion that Ganter Bridge is not considered as Extradosed Bridge, since the finback stay cable is not subjected to cable vibration and its associated fatigue issue. Similar bridge type is being constructed in Tampa, Florida, USA by Tampa Hillsborough Transit Authority for toll bridge as shown in Figure 5. Instead of two parallel finbacks as in the case of Ganter Bridge, the West Selmon Bridge has a single finback.
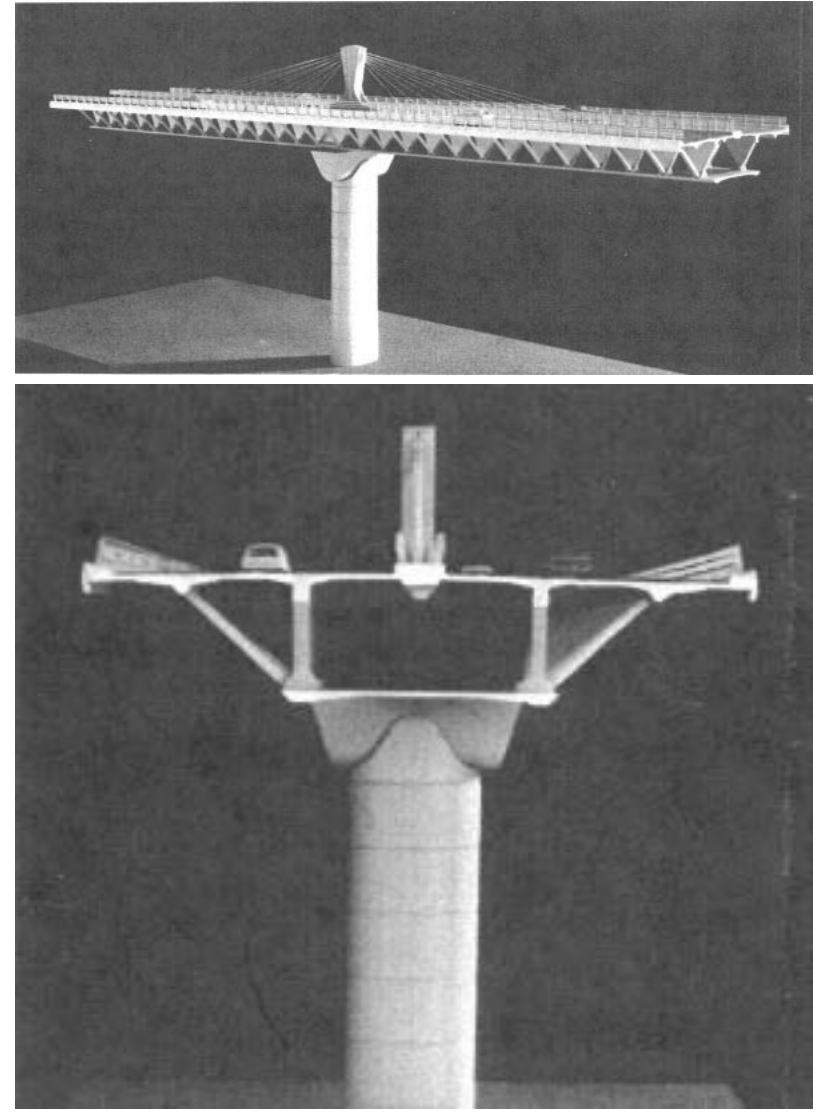

Figure 3. Proposed Autoroute A64 viaduct across ArretDarre, France - Isometric and Cross Section Views of the Model

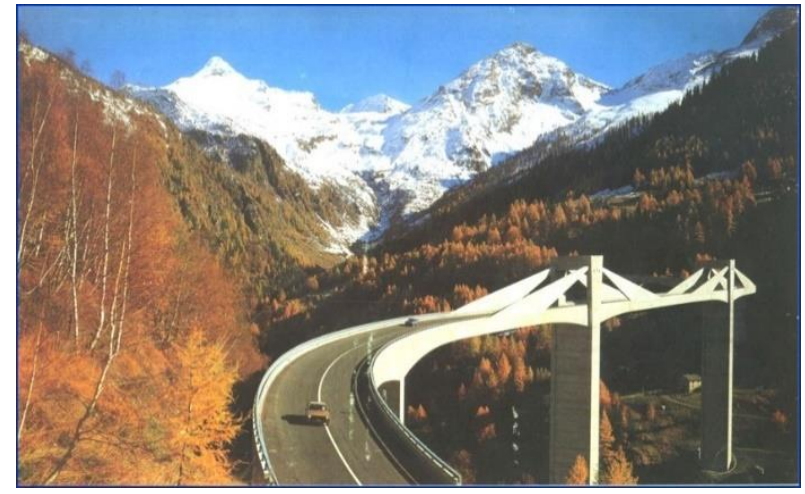

Figure 4. Ganter Bridge, Switzerland

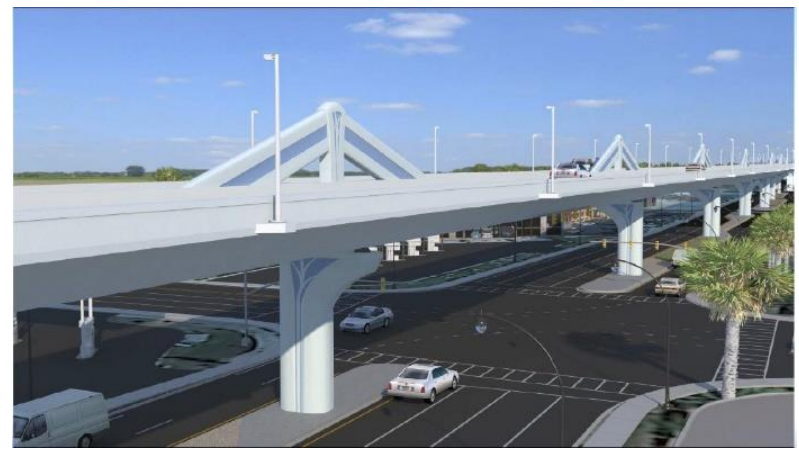

Figure 5. West Selmon Extension Viaduct Rendering, Tampa, Florida (courtesy of Kiewit-AECOM D/B Team) 


\section{What is Extradosed Bridge Concept?}

Extradosed bridge is a bridge concept which has a structural behavior and elements that combines both of a balanced cantilever bridge and a cable stayed bridge.

fib Bulletin 97 "External Tendons for Bridges" [4] (about to be published in 2021) defined conventional external tendons bridge is the bridge supported by external tendons fall into the depth limit of the supporting girder as shown in Figure 6. Therefore, bridges with external tendons outside the depth limit also falls into Extradosed bridges type as shown in Figure 7.

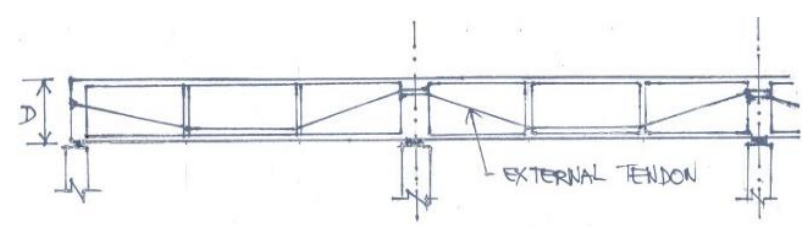

Figure 6. Conventional External Tendons Bridge

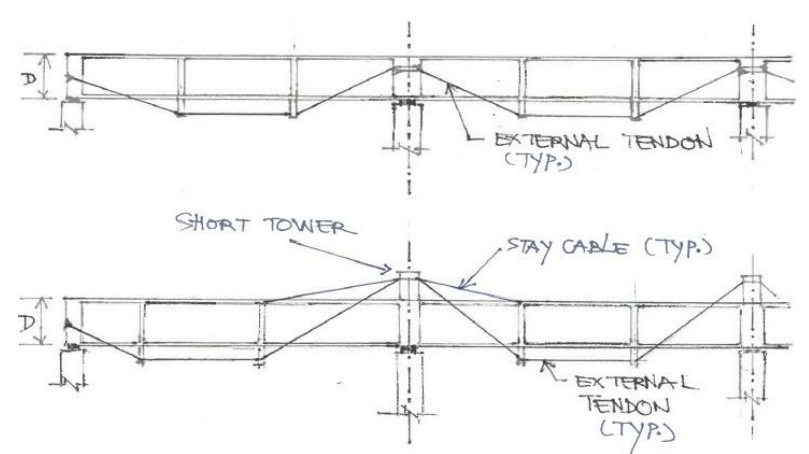

Figure 7. Other Forms of Extradosed Bridge

From structural design perspective one of the most attractive design features of Extradosed bridge is the stay cable allowable stresses at Service Limit State. In conventional Cable Stay bridges, the allowable stress of stay cable main tension element is about 0.4 $\mathrm{fpu}-0.45 \mathrm{fpu}$, while in Extradosed bridge stay cable is $0.55 \mathrm{fpu}-0.6 \mathrm{fpu}$. The $0.6 \mathrm{fpu}$ allowable stress is consistent with the internal tendon stress level after all long-term loss of prestresses at day 10,000 for conventional post-tensioned bridge.

Figure 8 provides the comparison of geometric parameters which define a typical Cable Stayed and Extradosed bridges [2]. These parameters are very important guidance for bridge engineers when designing these types of bridges during conceptual and preliminary design stages. The notable differences between the two types of bridge can be summarized in Table 1. One of the most important differences is the stay cables angle with reference to horizontal ( $\alpha$ ). It is obvious from Figure 8 that extradosed bridge has smaller stay cable angle in comparison with cable stayed bridge. Smaller angle a would result in smaller stress change $\Delta \sigma_{\mathrm{L}}$ in the stay cable, therefore smaller fatigue resistance requirement, unlike the main tension element for conventional cable stayed bridge. Stress change $\Delta \sigma_{\mathrm{L}}$ in main tension element determines the allowable stress and required fatigue resistance as shown in Table 2 and Figure 9. The main tension element vs stress change $\left(\Delta \sigma_{L}\right)$ criteria is the most important parameter in designing Extradosed bridges which first proposed by Japan Prestressed Concrete Association in 2000 [5] and later adopted by fib [6]. Extradosed bridges caught the attention of the international bridge engineering community after the first Extradosed bridge, Odawa Blueway Bridge, built in Japan in 1996 [7].
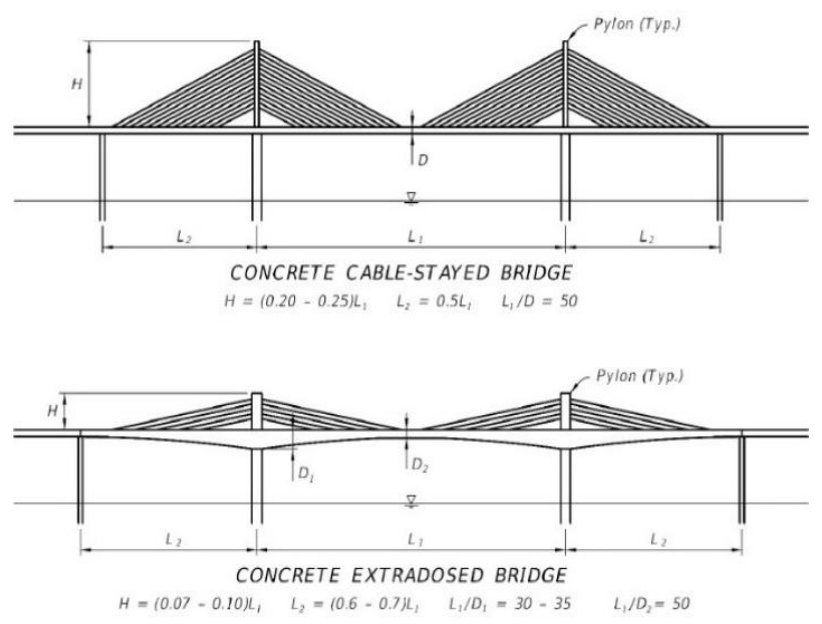

Figure 8. Typical Geometric Comparison between Cable Stayed and Extradosed Bridge [2]

Table 1. Brief Comparison between Cable Stayed and Extradosed Bridge

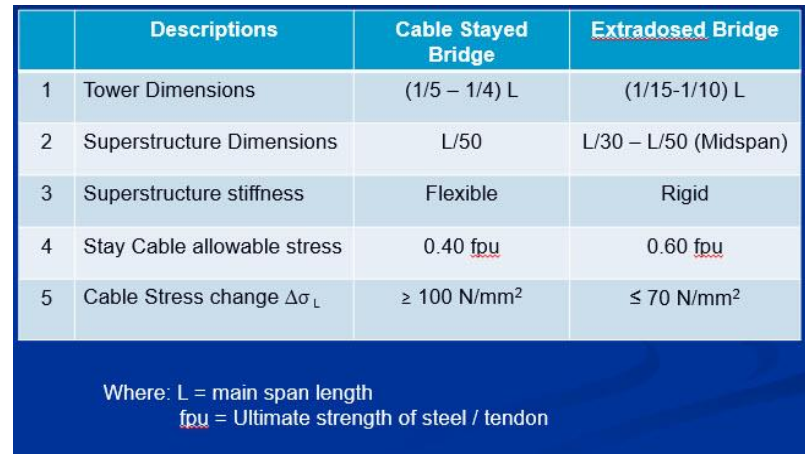

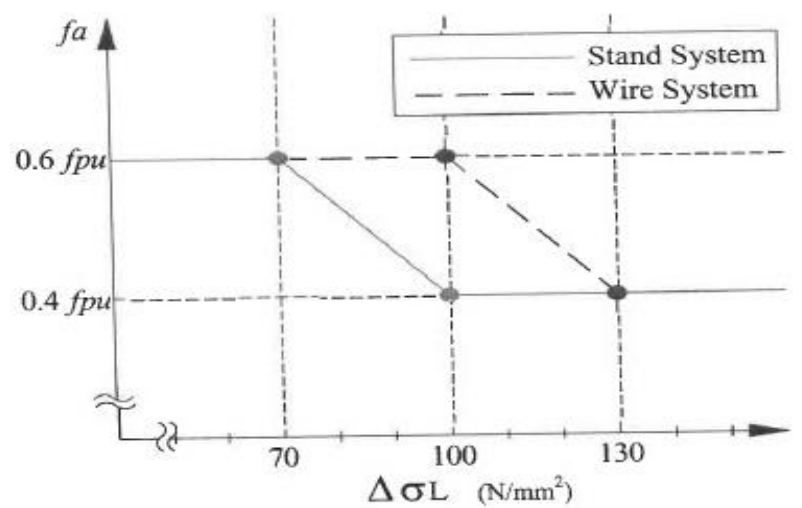

Figure 9. Steel Allowable Stress vs Stress Change [5,6] 
Table 2. Minimum Performance Requirement-S-N Values for Main Tension Elements and Cable System [6]

\begin{tabular}{|c|c|c|c|c|c|}
\hline & & & $k_{2}$ & Upper stress $\sigma_{\text {mat }}$ & $\begin{array}{l}\text { Stress range } \Delta \sigma \text { (MPa) } \\
\text { at } 2 \cdot 10^{\circ} \text { load cycles }\end{array}$ \\
\hline \multirow{5}{*}{ WIRE } & A & $\approx 6^{n}$ & 8 & $45 \%$ AUTS & 370 \\
\hline & $B_{\text {ancent }}{ }^{3}$ & 5 & 7 & $45 \%$ GUTS & 285 \\
\hline & $B_{\text {anissond }}{ }^{3 /}$ & 5 & 7 & $55 \%$ GUTS & 200 \\
\hline & $C_{\text {eay cotie }}$ & 4 & 6 & $45 \%$ GUTS & $200^{2}$ \\
\hline & $C_{\text {entabied }}$ & 4 & 6 & $55 \%$ GUTS & $140^{2}$ \\
\hline \multirow{5}{*}{ STRAND } & A & $\approx 6^{n}$ & 8 & $45 \%$ AUTS & 300 \\
\hline & B ingente & 5 & 7 & $45 \%$ GUTS & 250 \\
\hline & $B_{\text {otostonat }}$ & 5 & 7 & $55 \%$ GUTS & 180 \\
\hline & $C_{\text {warcose }}$ & 4 & 6 & $45 \%$ GUTS & $200^{21}$ \\
\hline & $C_{\text {ortotiod }}$ & 4 & 6 & $55 \%$ GUTS & $140^{2 i}$ \\
\hline
\end{tabular}

\section{Selected Extradosed bridges}

About eight years after Mathivat introduced the Extradosed bridge Concept in 1988, the first Extradosed bridge called Odawa Blueway Bridge was constructed in Japan in 1996 as shown in Figure 10. After this bridge was constructed, there are more than 30 other Extradosed bridges built in Japan [2].

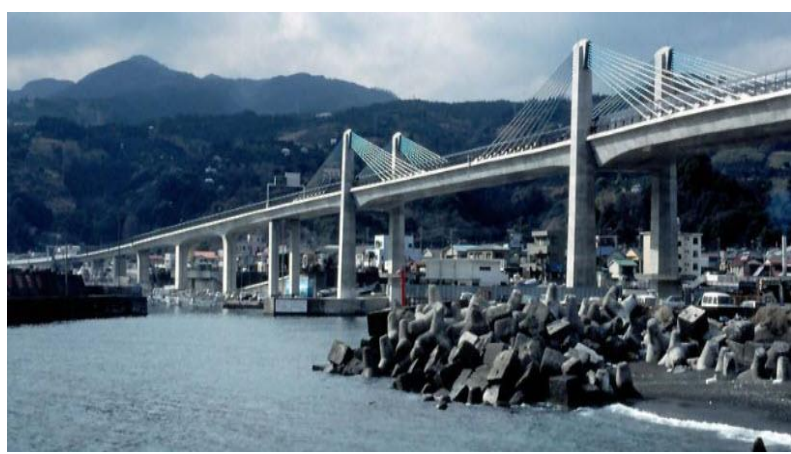

Figure 10. Odawa Blueway Bridge in Japan (Courtesy of Sumitomo Mitsui, Japan).

In the US, several Extradosed bridges have also been constructed. The first Extradosed bridge in US is Pearl Harbor Memorial Bridge North Bound constructed in 2012 as shown in Figure 11 and the South Bound was completed in 2015. The bridge also known as Q Bridge located in New Haven, Connecticut.

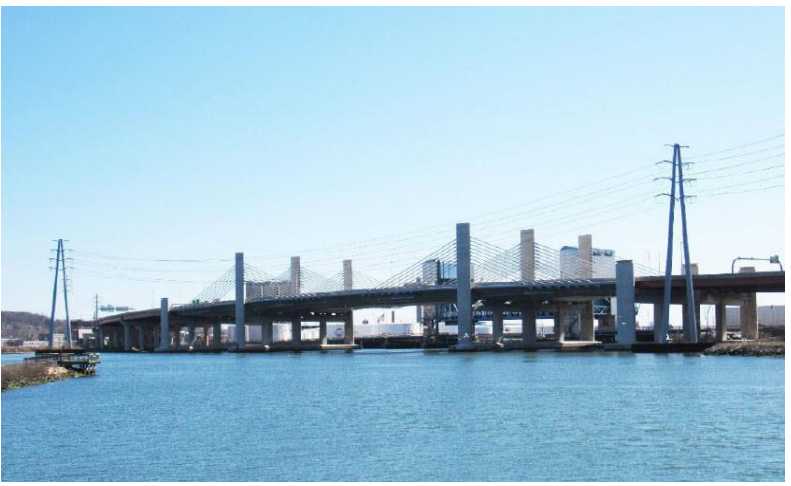

Figure 11. Pearl Harbor Bridge NB
In 2014 US constructed the second Extradosed bridge I-35 Brazos River Bridge in Waco, Texas. Unlike other Extradosed bridges constructed with posttensioned concrete, this bridge has steel girders superstructure.

In 2017 US constructed another Extradosed Bridge called St. Croix Crossing located partly in Still Water, Minnesota and St. Joseph, Wisconsin as shown in Figure 12.

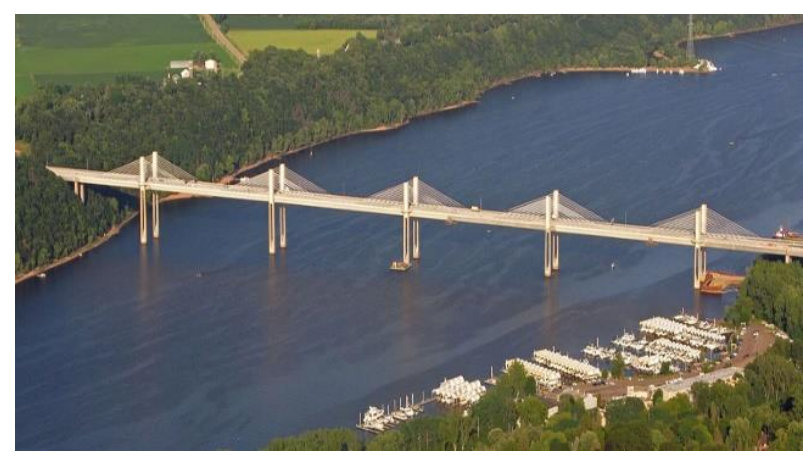

Figure 12. St. Croix Crossing Extradosed Bridge (Courtesy of Minnesota Department of Transportation)

\section{Closing}

The Extradosed bridge is an elegant and economical bridge type suitable for medium span ranges. The Extradosed bridge Concept came from a very simple idea utilizing external tendons and increased eccentricity for the benefit of increasing positive moment to counteract the negative bending moment at the critical sections over the pier column which is the largest negative moment during construction. This is an example of an innovation in bridge engineering born from a very simple idea in structural design. Hopefully, this short article will inspire young future innovators in bridge engineering.

\section{Disclaimer}

The opinion and recommendations expressed in this article are those of the author and not necessarily of the Florida Department of Transportation (FDOT) or Indonesian Civil Engineers' Society of North America (ICESNA).

\section{References}

1. Mathivat, J., The Cantilever Construction of Prestressed Concrete Bridges, John Wiley and Sons, 1979.

2. Stroh, S.L., Extradosed Prestressed Bridges, 2014 FDOT Design Training Expo, Orlando, FL.

3. Mathivat, J., Recent Development in Prestressed Concrete Bridges, FIP Notes, 1988

4. fib Bulletin 97 External Tendons for Bridges, Final Draft January 2021 (to be published in 2021). 
Theryo, T.S. / The Development of Extradosed Bridge Concept / CED, Vol. 23, No. 1 March 2021, pp. $62-66$

5. Standard Specification for Cable Stayed Bridge and Extradosed Bridge, Japan Prestressed Concrete Engineering Association, November 2000.
6. fib Bulletin 89 Acceptance of Cable Systems Using Prestressing Steels, 2019.

7. Kasuga, A., Extradosed Bridges in Japan, Fib Structural Concrete, 2006. 